\title{
Ensayo
}

(Essay)

\section{LA GENÉTICA COMO HERRAMIENTA PARA EL ESTUDIO Y CONSERVACIÓN DEL GÉNERO ALOUATTA EN MÉXICO}

\author{
LAURA Elisa ARGüELLO-SÁNCHEZ \& Luis MANUEl GARCÍA-FERIA* \\ Red de Biología y Conservación de Vertebrados. Instituto de Ecología, A. C. Carretera antigua a \\ Coatepec No. 351. El Haya. C.P. 91070. Xalapa, Veracruz, México. \\ *Correspondencia:<luizoo@yahoo.com>
}

Argüello-Sánchez, L. E. \& García-Feria, L. M. 2014. La genética como herramienta para el estudio y conservación del género Alouatta en México. Acta Zoológica Mexicana (n. s.), 30(2): 387-394.

RESUMEN. El estudio de la estructura genética de las poblaciones animales se ha convertido en una herramienta de gran valor en los programas de conservación de especies, particularmente de aquellas que se encuentran en peligro de extinción. Se han utilizado diferentes parámetros poblacionales, tanto en animales cautivos como silvestres, para evaluar su estado genético y su viabilidad a largo plazo. En México, diferentes herramientas genéticas se han usado para estudiar las tres especies de primates no humanos a fin de fundamentar o comprobar diversos aspectos biológicos establecidos a través de observaciones y trabajos de campo. Entre los aspectos de esas especies que se han estudiado desde una perspectiva genética se incluyen varias cuestiones taxonómicas y biogeográficas, la estructura y diferenciación de las poblaciones dentro del área de distribución, los patrones de parentesco y sistemas sociales, entre otros. Estos estudios han conducido a una mejor comprensión de la biología de esos primates, y al desarrollo de estrategias más efectivas para la conservación y supervivencia del género Alouatta en México.

Palabras clave: Alouatta palliata, Alouatta pigra, genética, conservación.

Argüello-Sánchez, L. E. \& García-Feria, L. M. 2014. Genetics as a tool for the study and conservation of the genus Alouatta in Mexico. Acta Zoológica Mexicana (n. s.), 30(2): 387-394.

ABSTRACT. The genetic study of animal populations has become a valuable tool in conservation programs, particularly of those species that are endangered. Different population parameters can be determined in both captive and wild animals to assess their genetic status and long-term viability. In Mexico, different genetic tools have been used in the three species of nonhuman primates to assess, reinforce or test different biological aspects that have been determined by observations and field work. Among others, taxonomic and biogeographic aspects, differentiation and population structures, patterns of kinship and social systems have been made to know and understand the biology of the species and direct conservation and survival strategies of the genus Alouatta in Mexico.

Key words: Alouatta palliata, Alouatta pigra, genetic, conservation.

Recibido: 08/11/2013; aceptado: 13/03/2014. 


\section{LA GENÉTICA EN EL ESTUDIO DE FAUNA SILVESTRE}

El desarrollo de nuevos tipos de marcadores genéticos así como la mejora en los métodos analíticos y la automatización de los análisis genéticos (Sunnucks 2000; Deyoung et al. 2005), ha vuelto factible y más generalizada la integración de métodos genéticos en los estudios de poblaciones de fauna silvestre (Deyoung et al. 2005). En la actualidad, una amplia variedad de cuestiones ecológicas pueden ser resueltas mediante el uso de técnicas de genética molecular (Burke 1994; Sunnucks 2000). Entre las aplicaciones más frecuentes de la genética en el campo de la biología de especies se encuentran la determinación del sexo o la identificación especifica de los individuos, la evaluación de la diversidad genética y la estructura poblacional, la determinación del parentesco, proporcionar el fundamento biológico el sistema de apareamiento, la estimación de la dispersión, migración y abundancia de individuos de una especie en un espacio y tiempo definidos, la historia demográfica poblacional, la detección de hibridación y la definición de unidades de manejo (Deyoung et al. 2005; Waits \& Paetkau 2005).

Por otro lado, gracias a la eficiencia del muestreo no invasivo (ej. pelo, excretas, plumas, saliva y cáscaras de huevo) para la obtención de material genético, ha sido posible el estudio genético de especies crípticas, raras o en peligro de extinción (Waits \& Paetkau 2005) de las cuales se conocía muy poco. En el caso de los primates no humanos, el uso del muestreo no invasivo para estudios genéticos de poblaciones silvestres ha sido crucial, pues la gran mayoría de las especies viven en selvas densas y tienden a ser huidizas, dificultando su observación. Las muestras de ADN obtenidas de forma no invasiva pueden ser analizadas con una amplia gama de marcadores moleculares. Comúnmente se utilizan ADN mitocondrial, microsatélites, los polimorfismos en la longitud de fragmentos amplificados (AFLP) y diferencias en el complejo principal de histocompatibilidad (MHC) (Ferreira da Silva et al. 2012). El uso de esos marcadores moleculares ha permitido responder una vasta cantidad de preguntas de investigación.

\section{GENÉTICA DE LA CONSERVACIÓN}

El enfoque de la genética en la vida silvestre tomó gran fuerza a partir de las investigaciones pioneras de O'Brien et al. (1983) con guepardos (Acinonyx jubatus), las cuales proporcionaron información importante para entender las notables alteraciones fisiológicas observadas en la especie (Menotti-Raymond \& O’Brien 1993). Estos hallazgos llevaron a realizar estudios similares en un número cada vez mayor de especies en vida libre y en cautiverio, lo que ha contribuido a resolver diversos problemas taxonómicos, a determinar el riesgo de enfermedades, a explicar algunos problemas reproductivos y, lo que es muy importante, a sugerir programas de manejo y estrategias de conservación de las especies a largo plazo (Cohn 1990). Si bien no 
se recomienda considerar únicamente los resultados de estudios genéticos para la planeación de los programas de conservación de las especies, la combinación de sus datos con los obtenidos en estudios tradicionales pueden proveer bases más sólidas para desarrollar estrategias de conservación efectivas (Avise \& Hamrick 1996).

De acuerdo con Frankham (1995), existen varios temas genéticos que son de particular relevancia para la conservación biológica, entre los que se encuentran los siguientes: la depresión de la variabilidad por endogamia, la pérdida de variación genética en poblaciones pequeñas, la fragmentación de las poblaciones y la consecuente reducción de la migración. Debido a su importancia, estos temas son comúnmente abordados en los estudios de las especies de primates bajo algún tipo de riesgo.

\section{ESTUDIOS GENÉTICOS DEL GÉNERO ALOUATTA EN MÉXICO Y SU APORTE A LA CONSERVACIÓN}

Con la severa deforestación de los bosques tropicales en los últimos 60 años, junto con la caza excesiva y el comercio ilegal de mascotas, las poblaciones de las dos especies de monos aulladores de México (Alouatta palliata y Alouatta pigra) han sido drásticamente disminuidas (Estrada \& Coates-Estrada 1994; Sánchez et al. 1998; Duarte-Quiroga \& Estrada 2003), siendo A. palliata la que presenta mayor reducción de sus poblaciones con cerca del 90\% (Zarza-Villanueva 2006; Rodríguez-Luna et al. 2009). Es por ello que ambas especies están consideradas como especies en peligro de extinción por la Nom-059-Semarnat-2010 y clasificadas por la Unión Internacional para la Conservación de la Naturaleza (IUCN por sus siglas en inglés) como "En peligro", en el caso de Alouatta pigra, y "Críticamente en peligro", en el caso de Alouatta palliata ssp. mexicana (Cuarón et al. 2008; Marsh et al. 2008). Debido al riesgo en que se encuentran estas especies, se están creando programas para su conservación a nivel nacional (Oropeza-Hernández \& Rendón-Hernández 2012) dentro de los que se contempla la generación de información que permita tener un panorama más completo de los aspectos ecológicos, conductuales, fisiológicos y genéticos de estas especies para la toma de decisiones en los planes de acción.

Los estudios con primates silvestres mexicanos fueron iniciados en 1975 por investigadores adscritos a la Estación de Biología Tropical "Los Tuxtlas" de la Universidad Nacional Autónoma de México (UNAM), en el Estado de Veracruz, donde se encuentran las especies Alouatta palliata y Ateles geoffroyi. Posteriormente se fueron integrando un gran número de investigadores a este campo de la primatología en México, lo cual se ha traducido en numerosos estudios de la distribución de las poblaciones de las especies mencionadas, su demografía, las interacciones sociales y conducta reproductiva; el efecto de las presiones antropogénicas en la demografía, conducta y su distribución; patrones de movimiento, entre muchos otros temas (ej. Estrada 1982; Jimenez 1992; Estrada \& Coates-Estrada 1994; García-Orduña 1996; 
Estrada et al. 1999; Serio-Silva \& Rico-Gray 2000; Duarte-Quiroga \& Estrada 2003; Pozo-Montuy 2003; Van Belle \& Estrada 2005; Pozo-Montuy \& Serio-Silva 2007; Ramírez-Julian 2010).

No obstante, los estudios genéticos de Alouatta palliata y Alouatta pigra comenzaron a realizarse hace poco más de una década atrás. Los primeros estudios que incluyeron muestras de individuos de poblaciones las poblaciones mexicanas fueron los realizados por Mudry et al. (2001), Cortes-Ortiz et al. (2003) y Ellsworth \& Hoelzer (2006). En el primero de esos estudios, en un proyecto de citogenética con atélidos se utilizaron muestras de A. palliata y Ateles geoffroyi. . El segundo fue un estudio de sistemática molecular y filogeografía del género Alouatta, en el que se utilizaron marcadores mitocondriales y nucleares para realizar un análisis biogeográfico-histórico para poder inferir las relaciones filogenéticas entre nueve de las diez especies reconocidas del género. El tercero y último tuvo como objetivo dilucidar la biogeografía histórica de los monos aulladores en Centroamérica, buscando evidencia genética que apoyara la hipótesis de la invasión de un ancestro común proveniente de Sudamérica, que migró hasta llegar al sur de México. Estos primeros estudios, si bien no fueron enfocados únicamente a los primates mexicanos, permitieron responder preguntas relevantes a nivel de género y, lo que es más importante, sentaron las bases para la realización de estudios futuros.

Un problema frecuente para la elaboración de programas de conservación eficaces ha sido la delimitación de especies o unidades evolutivas independientes (Domínguez-Domínguez \& Vázquez-Domínguez 2009). En este aspecto, el estudio de sistemática molecular de Cortés-Ortiz (2003) representó una importante contribución, al encontrar un 5.7\% de diferencias significativas a nivel de ADN mitocondrial entre A. palliata y A. pigra, brindando así evidencia genética de diferenciación entre esas especies. Originalmente, A. pigra fue descrita por Lawrence (1933) como dos subespecies de A. palliata: 1) A. palliata pigra en la península de Yucatán y norte de Guatemala y 2) A. palliata luctuosa en Belice (Leopold 1959). Tiempo después estas subespecies fueron reclasificadas como dos variantes de una nueva especie (Smith 1970), sobre la base de diferencias conductuales y anatómicas a las que posteriormente se les sumó la evidencia genética.

Desde la propuesta de la separación específica de A. pigra de A. palliata, se supo de la existencia de una zona de simpatría de estas especies en la región de Macuspana, Tabasco (Brandon-Jones 2006). En esta región se observaron tropas con individuos de A. palliata y A. pigra (basado en caracteres morfológicos), así como de individuos con características de ambas especies. La existencia de hibridación entre las dos especies fue después confirmada con evidencia genética y comparaciones morfológicas (Cortés-Ortiz et al. 2007; Kelaita \& Cortés-Ortiz 2013).

Entre los estudios de genética poblacional realizados exclusivamente para las especies de primates no humanos mexicanos, figuran dos para A. pigra (García del 
Valle et al. 2005; Améndola 2009) y uno para A. palliata (Argüello-Sánchez 2012). A grandes rasgos, estos trabajos se enfocaron en comparar el nivel de variación genética de poblaciones, tanto en hábitat conservado como en hábitat perturbado. Los datos obtenidos en esos estudios han permitido determinar cuáles de las poblaciones estudiadas presentan la mayor variación genética y cuales contienen variantes genéticas únicas, lo que ayuda a la determinación de áreas prioritarias para la conservación de las especies. Por otra parte, el análisis filogenético realizado en uno de estos estudios, reveló la presencia de dos linajes de origen materno (Argüello-Sánchez 2012), información que debe ser considerada al establecer prioridades de conservación (Rojas 2003). Es importante también no enfocarse tan solo en la conservación de las especies, sino considerar las unidades evolutivas dentro de ellas para preservar los procesos evolutivos que son indispensables para la permanencia de las especies y sus hábitats a largo plazo (Crandall et al. 2000; Domínguez-Domínguez \& Vázquez-Domínguez 2009).

Recientemente se realizaron algunos estudios genéticos para evaluar los patrones de parentesco entre los miembros de los grupos sociales en ambas especies de monos aulladores (Van Belle et al. 2012; Baiz 2013), lo cual ha permitido adquirir un conocimiento más claro del sistema social y la dispersión de ambas especies de Alouatta. Siendo estos primates organismos longevos, es importante considerar el desarrollo de estudios tradicionales (ej. Crockett \& Elisenberg 1987) de muy largo plazo para obtener datos similares de la dispersión de individuos, y aunque serian menos precisos que los estudios genéticos, la combinación de ambos tipos de estudios podían generar información muy robusta (ej. Pope 1992). Así el conocer el sistema social y la dispersión de las especies, ayudará a entender y predecir la forma en que se distribuye la variación genética entre y dentro de las poblaciones (Pope 1992; Storz 1999).

La contribución de los estudios antes mencionados a la generación de conocimiento ha sido la base para preservación de los primates del género Alouatta, ya que desempeñan un papel importante en la conservación y regeneración de las selvas al dispersar las semillas de muchas plantas (Chapman \& Russo 2007). Sin embargo, persisten muchas interrogantes que podrían ser contestadas por medio de estudios genéticos. Por ejemplo, se necesita ampliar los estudios de genética poblacional a lo largo de las áreas de distribución de las especies y obtener así un panorama más amplio de la variación genética existente. Tener esta información facilitaría la ubicación del origen de los individuos decomisados y recuperados del tráfico ilegal de especies, y permitiría además una mejor planeación de programas de translocación de individuos o tropas en inminente riesgo, intentando mantener el linaje y composición genética naturales de cada región. Así mismo, se debería considerar la evaluación de genes que están implicados en la resistencia a patógenos (ej. complejo principal de histocompatibilidad) u otros genes que se relacionen directa o indirectamente con la adecuación de las especies. 
AGRADECIMIENTOS. Agradecemos al proyecto (No. 108865, FOMIX-VERACRUZ CONACYT) "Consecuencias de la perturbación del hábitat sobre la variabilidad y estructura genéticas de poblaciones de Alouatta palliata mexicana: una especie de primate en peligro de extinción en Veracruz, México"; Al Dr. Juan Carlos Serio-Silva por sus sugerencias y revisión al escrito.

\section{LITERATURA CITADA}

Améndola, M. 2009. Estudio de la variabilidad genética en poblaciones de Alouatta pigra del estado de Campeche: implicaciones para la conservación. Tesis de Doctorado. Instituto de Ecología, A. C.

Argüello-Sánchez, L. E. 2012. Genética de la conservación en Alouatta palliata mexicana: evaluación del efecto de la fragmentación del hábitat y sus poblaciones en Veracruz. Tesis de Maestría. Instituto de Ecología, A. C., Xalapa, Ver.

Avise, J. C. \& Hamrick, J. L.. 1996. Conservation genetics: case histories from nature. Chapman \& Hall, New York.

Baiz, M. 2013. Intragroup genetic relatedness in two howler monkey species (Alouatta pigra and A. palliata): Implications for understanding social systems and dispersal. Master Degree. The University of Michigan.

Brandon-Jones, D. 2006. Apparent Confirmation that Alouatta villosa (Gray, 1845) is a Senior Synonym of A. pigra Lawrence, 1933 as the Species-Group Name for the Black Howler Monkey of Belize, Guatemala and Mexico. Primate Conservation, 21: 41-43.

Burke, T. 1994. Spots before the eyes: molecular ecology. Trends in Ecology \& Evolution, 9: 355357.

Chapman, C. A. \& Russo, S. E. 2007. Primate seed dispersal: Linking behavioral ecology with forest community structure, pp. 510-525. In: C. Campbell, A. Fuentes, A. MacKinnon, S. Bearder and R. Stumpf (Eds.). Primates in Perspective. Oxford University Press, New York.

Cohn, J. 1990. Genetics for wildlife conservation. BioScience, 40: 167-171.

Cortes-Ortiz, L., Bermingham, E., Rico, C., Rodriguez-Luna, E., Sampaio, I. \& Ruiz-Garcia, M. 2003. Molecular systematics and biogeography of the Neotropical monkey genus, Alouatta. Molecular Phylogenetics and Evolution, 26: 64-81.

Cortés-Ortiz, L., Duda, T. F., Canales-Espinosa, D., García-Orduña, F., Rodríguez-Luna, E. \& Bermingham, E. 2007. Hybridization in Large-Bodied New World Primates. Genetics, 176: 2421 2425.

Crandall, K. A., Bininda-Emonds, O. R. P., Mace, G. M. \& Wayne, R. K. 2000. Considering evolutionary processes in conservation biology. Trends in Ecology \& Evolution, 15: 290-295.

Crockett, C. M. \& EIisenberg, J. F. 1987. Howlers: Variations in group size and demography. In: B. Smuts, D. Cheney, R. Seyfarth, R. Wrangham and T. Struhsaker (Eds.). Primate Societies. University of Chicago Press Chicago, IL USA.

Cuarón, A. D., Shedden, A., Rodríguez-Luna, E., de Grammont, P. C. \& Link, A. 2008. Alouatta palliata ssp. mexicana. IUCN Red List of Threatened Species, www.iucnredlist.org.

Deyoung, R. W., Honeycutt, R. L. \& Brennan. 2005. The molecular toolbox: genetic techniques in wildlife ecology and management. Journal of Wildlife Management, 69: 1362-1384.

Domínguez-Domínguez, O. \& Vázquez-Domínguez, E. 2009. Filogeografía: aplicaciones en taxonomía y conservación. Animal Biodiversity and Conservation, 62: 53-60.

Duarte-Quiroga, A. \& Estrada, A. 2003. Primates as pets in Mexico City: An assessment of the species involved, source of origin, and general aspects of treatment. American Journal of Primatology, 61: 53-60.

Ellsworth, J. \& Hoelzer, G. 2006. Genetic evidence on the historical biogeography of Central American howler monkeys. In: L. S. and F. J. (Eds.). Primate Biogeography. Springer, New York. 
Estrada, A. 1982. Survey and census of howler monkeys (Alouatta palliata) in the rain forest of "Los Tuxtlas," Veracruz, Mexico. American Journal of Primatology, 2: 363-372.

Estrada, A., Anzures, A. \& Coates-Estrada, R. 1999. Tropical rain forest fragmentation, howler monkeys (Alouatta palliata), and dung beetles at Los Tuxtlas, Mexico. American Journal of Primatology, 48: 253-262.

Estrada, A. \& Coates-Estrada, R. 1994. La contracción y fragmentación de las selvas y las poblaciones de primates silvestres: el caso de Los Tuxtlas, Veracruz. La Ciencia y el Hombre, 18: 45-70.

Ferreira da Silva, M. J., Minhos, T., Sa, R. M. \& Bruford, M. W. 2012. Using Genetics as a Tool in Primate Conservation. Nature Education Knowledge, 3: 89.

Frankham, R. 1995. Effective population size/adult population size ratios in wildlife: a review. Genetics Research, 66: 95-107.

García del Valle, Y., Estrada, A., Espinoza, E., Lorenzo, C. \& Naranjo, E. 2005 Genética de poblaciones de monos aulladores (Alouatta pigra) en hábitat continuo y fragmentado en la Selva Lacandona, México: un estudio preliminar. Universidad y Ciencia, 2: 55-60.

García-Orduña, F. 1996. Distribución y abundancia del mono aullador Alouatta palliata y el mono araña Ateles geoffroyi en fragmentos de selva del municipio de San Pedro Soteapan, Veracruz. Tesis Licenciatura. Universidad Veracruzana, Xalapa, Veracruz.

Jimenez, H. J. 1992. Distribución y abundancia del recurso alimenticio en un fragmento de selva alta perennifolia y su uso por Ateles y Alouatta en el ejido Magallanes (Municipio de Soteapan, Veracruz). Tesis de Licenciatura. Universidad Veracruzana, Xalapa, Veracruz.

Kelaita, M. A. \& Cortés-Ortiz, L. 2013. Morphological variation of genetically confirmed Alouatta pigra $\times$ A. palliata hybrids from a natural hybrid zone in Tabasco, Mexico. American Journal of Physical Anthropology, 150: 223-234.

Lawrence, B. 1933. Howler monkeys of the palliata group. Bulletin of the Museum of Comparative Zoology at Harvard College, 75: 313 -354.

Leopold, A. S. 1959. Wildlife of Mexico. University of California Press, Berkeley, California.

Marsh, L. K., Cuarón, A. D., Cortés-Ortiz, L., Shedden, A., Rodríguez-Luna, E. \& de Grammont, P. C. 2008. Alouatta pigra. IUCN Red List of Threatened Species.

Menotti-Raymond, M. \& O'Brien, S. J. 1993. Dating the genetic bottleneck of the African cheetah. Proceedings of the National Academy of Sciences, 90: 3172-3176.

Mudry, M. D., Rahn, I. M. \& Solari, A. J. 2001. Meiosis and chromosome painting of sex chromosome systems in Ceboidea. American Journal of Primatology, 54: 65-78.

O'Brien, S. J., Wildt, D. E., Goldman, D., Merril, C. R. \& Bush, M. 1983. The cheetah is depauperate in genetic variation. Science, 221: 459-462.

Oropeza-Hernández, P. \& Rendón-Hernández, E. 2012. Programa de Acción para la Conservación de las Especies: Primates, Mono Araña (Ateles geoffroyi) y Monos Aulladores (Alouatta palliata, Alouatta pigra). Secretaría de Medio Ambiente y Recursos Naturales / Comisión Nacional de Áreas Naturales Protegidas, México.

Pope, T. 1992. The influence of dispersal patterns and mating system on genetic differenciation within and between populations in the red howler monkey (Alouatta seniculus). Evolution, 42: 11121128.

Pozo-Montuy, G. 2003. Comportamiento de monos aulladores (Alouatta pigra) en hábitat fragmentado, en la ranchería Leona Vicario, Balancán, Tabasco. Tesis de Licenciatura. Universidad Autónoma de Tabasco, Villahermosa, Tabasco.

Pozo-Montuy, G. \& Serio-Silva, J. 2007. Movement and resource use by a group of Alouatta pigra in a forest fragment in Balancán, México. Primates, 48: 102-107. 
Ramírez-Julian, R. 2010. Respuestas conductuales de monos aulladores negros, Alouatta pigra, viviendo en remanentes de vegetación entre pastizales en Balancán, Tabasco. Tesis de Maestría. Instituto de Ecología, A.C. , Xalapa, Veracruz.

Rodríguez-Luna, E., Solórzano-García, B., Shedden, A., Rangel-Negrín, A., Dias, P. A. D., Cristóbal-Azkárate, J., Cortés-Ortiz, L., Dunn, J. C., Domingo-Balcells, C., Sánchez, S., Vea-Baró, J. \& Cornejo, J. 2009. Taller de Conservación, Análisis y Manejo Planificado para los primates mexicanos, 2006. Universidad Veracruzana.

Rojas, M. 1992. The species problem and conservation: What are we protecting? Conservation Biology, 6: $170-178$.

Serio-Silva, J. C. \& Rico-Gray, V. 2000. Use of a stream by Mexican howler monkeys. The Southwestern Naturalist, 52: 332-333.

Smith, J. D. 1970. The systematic status of the black howler monkey, Alouatta pigra Lawrence. Journal of Mammalogy, 51: 358-369.

Storz, J. 1999. Genetic consequences of mammalian social structure. Jay F. Storz Publications. Papers in Biological Science

Sunnucks, P. 2000. Efficient genetic markers for population biology. Trends in Ecology \& Evolution, 15: 199-203.

Sánchez, O., Pineda, M., Benítez, H., González, B. \& Berlanga, H. 1998. Guía de identificación para aves y mamíferos silvestres de mayor comercio en México protegidos por la C.I.T.E.S. . Secretaría de Medio Ambiente, Recursos Naturales y Pesca/Comisión Nacional para el Conocimiento y Uso de la Biodiversidad México, D.F.

Van Belle, S. \& Estrada, A. 2005. Cambios demográficos en poblaciones de monos aullador negro (Alouatta pigra) como consecuencia de la fragmentación del hábitat. Universidad y Ciencia, 2: 1-9.

Van Belle, S., Estrada, A., Strier, K. B. \& Di Fiore, A. 2012. Genetic structure and kinship patterns in a population of black howler monkeys, Alouatta pigra, at Palenque National Park, Mexico. American Journal of Primatology, 74:948-957.

Waits, L. P. \& Paetkau, D. 2005. Noninvasive genetic sampling tools for wildlife biologists:a review of applications and recommendations for acurate data collection. The Journal of Wildlife Management, 69: 1419-1433.

Zarza-Villanueva, H. 2006. Ficha técnica de Alouatta palliata, pp. 7. In: R. Medellín (Ed.). Los mamíferos mexicanos en riesgo de extinción según el PROY-NOM-059-ECOL-2000. Instituto de Ecología, Universidad Nacional Autónoma de México, México, D.F. 